intic blue line on the gums, the whole muscular structure was wasted from the scapulse to the forearms, with a clammy coldnese, and a genersal want of power (feeling remaining perfect), and a total absence of spasm or contraction. The left hand was colder than the right, and slightly arched on its dorsal aspect; the thumb was wasted, and arched towards the palm.t

It was clearly one of those cases in which, as I pointed out in my work on the Bath waters, an error in diagnosis might easily be made; and to make sure of my own, I tested the urine of my patient, and found sulphuret of lead with the hydrosulphuret of ammonia, a test well known for its extreme accuracy, and the facility with which it may be applied to suspected fluids. With my concurrence, my patient transmitted, twelre days after leaving home, ten ounces of urine, and two bottles, each containing the same quantity of his usual drinking water, the one boiled, and the other fresh, to Mr. Herapath, whose report I subjoin :-

"I have analysed the contents of the three bottles forwarded, and I have found lead in all three of them. In the water from the boiler, there is also a trace of iron. Lead is in such quantity in the two waters, that $I$ have no doubt that they will produce symptoms of lead poisoning, perhaps in numbness, and stiffness of the extremities, or in 'lead colic', loss of appetite, constipation, atrophy, palsy, or paralysis, in fact, it is difficult to say in which form its effects will appear. The trace in the urine is very slight."

REMARKS. I believe this to be the first case on record in which chemistry has been applied to, by an analysis of the urine, to confirm a physical diagnosis in a case of palsy; it was highly important that this course should be adopted, because-from the variety of opinions formed of the nature of the case, the absence of many of the recognised symptoms of palsy from lead, the non-existence of the peculiar colic, and the age and great mental occupation of the patient at the period of invasion-the suspicion that lead was the cause of the mischief was not entertained, although other persons in the locality who had partaken of the same water had the ordinary and easily recognised forms of lead disease.

Tanquerel, in his highly interesting treatise upon Lead Diseases mentions one hundred and two cases of lead paralysis, of which forty were not preceded by colic: a fact worthy of observation in reference to the present case. I conceive that where a gradual loss of power takes place in the upper extremities, with a symmetrical loss of substance, without any impairing of the sensibility of the limbs, or any evidence of cerebral mischicf, we should look to lead, or some other mineral, as the cause of the disease, and I am inclined to think that the term paralysis has been wrongly applied to these cases. I would restrict paralysis solely to express a disease having its origin either in the brain or spinal marrow, and apply the term atrophy to those cases in which there is loss of power with loss of substance, and which are distinct in their invasion and seat from the true paralytic affections. This is a point which has not been sufficiently insisted upon by systematic writers; and hence the difficulty of diagnosis in cases which (except where the profession of the patient points to the cause of the disease) embarrass the practitioner in the formation of a correct diagnosis. In lead atrophy, the loss of power is dependent upon want of muscular structure, caused by gradual absorption, or rather by the want of healthy nutrition, producing an atonic condition of the nerves of motion; hence its invasion, unlike true paralysis, is gradual instead of sudden; the limbs are flaccid instead of spasmodically contracted, and remain very much in the same condition both night and day : in genuine hemiplegia the hand may at times relax from spasmodic closure, or vice versî́; while, in atrophy from lead, no such phenomena are observed. Hence, in the disease now under

+ The limbs are pendant on the side of the body; if raised, they fall like Inort masses, obeying the law of gravitation. In consequence of the permanent contraction of the pronacor muscles, and especially of the aterors, hand becomes rouuded, and the back of the hand arched. (Tanquereh) consideration, the whole of the natural motions of a limb may be produced b5 the assistance of the hand of an attendant, while in genuine paralysis they cannot be in any degree simulated; hence, wo hare a good diagnosis of a disease of rare occurrence, except among particular trades, and even more rare in the individual experience of members of the profession.

I have had a large experience in the treatment of the chronic form of lead atrophy, having had under my care, since I came to Bath, 236 cases, of whom 219 were either cured or greatly relieved by the Bath thermal treatment. The treatment of my patient consisted of thermal baths, electricity, and frictions, under which he improved, when, at the end of six weeks, his leave having expired, he returned to duty, shortly after which he was attacked with the furuncular epidemic, so that I am unable to state the progress of his case since March 19th, which I had hoped to do ; for cases like this generally require some considerable time before any great symptoms of amendment are visible.

I have only to remark, in conclusion, that where lead, in minute quantities, exists in the ordinary water drank by the patient, the gradual invasion of the symptoms may mislead the most careful practitioner. Lead appears, in these cases, gradually to poison the blood, without producing any of the symptoms of colic; indeed, Tanquere observes, that "when lead preparations have been introduced and absorbed in the system, they may show their deleterious influence directly by encephalopathy, an apyretic neuralgia of the encephalon", and producing symptoms of mental alienation, mislead the practitioner. Among the lead diseases he enumerates, are found arthralgy, paralysis, and encephalopathy, each of which may occur independently of the more recognised form of colic. Thus I have, I think, written enough at present to show that a number of diseases, preserving their individual characteristics, may put on appearances which may mislead even the best men in the profession, unaccustomed to observe them as directly arising from the introduction of lead into the alimentary canal.

\section{ANEURISM OF THE THORACIC AORTA.}

By JoHN WAI.sh, Esq., Manchester.

Case. C. D., aged 35 years, a tailor, consulted me on the 10th of last March, and gave me the following history of his case. Until twelve months ago he enjoyed perfect health; about that time he was attacked in the street by some ruffians, and, after a severe struggle, knocked down and badly beaten; he has never felt the same man since. Six months after this, he began to be troubled with a pain in the centre of the breast, also a harsh dry cough, and occasionally palpitation of the heart; latterly he has fits of shortness of breath when much hurricd; appetite always good; sleep very short and troubled; he has lost flesh, but was always thin; he has been treated most of the time by the surgeon of the society of which he is a member, and has been also a patient of the Royal Infirmary. He was told his disease was consumption, and the remedies employed consisted of cough mixtures, chest plasters, and cod-liver oil; nothing has done him any good. He wished to know from me whether there was any possibility of curing him; if there was, he would place himself under my care; but if not, he should prefer remaining under his own surgeon. I made a moderately careful examination of the case. I found the man sallow and cachectic in appearance; pulse quiet and regular; tongue rather foul; the apices of both lungs sounded clear on percussion, and the respiratory murmur was pure in the same parts; sounds of heart quite normal, and action regular.

Being satisfied that there was neither phthisis ncr disease of the heart present, I was disposed to regard the case as one of dyspepsia; and stating my views to the patient and his brother, who accompanied him, I prescribed in accordance with this opinion, and told the man to call again 
in a woek. He did so, and reported himself as nothing better. The pain-deep within the chest-was very severe, the cough very troublesome, and without any expectoration, and fits of dyspnoes more frequent and distressing. I again prescribed, endeavouring to relieve the thoracic symptoms (by leeching the chest, etc.), although a more extended stethoscopic examination failed to elicit any positive evidence of disease in that region.

On next seeing the patient, he informed me that he was decidedly worse in every respect. I now felt convinced that there was something wrong in the chest, which had hitherto escaped detection, and was determined, if possible, to find it out. I had the man stripped, and commenced my examination by carefully percussing the entire surface of the thorax. I discovered well marked, but by no means absolute dulness in a spot at the upper part of the sternum, corresponding to the second intercostal space, and covering a circle of about two inches in diameter. Orer this spot the heart sounds (or sounds exactly similar) were heard very loud; there was no impulse, nor any kind of bruit; between the scapula to the left of the spine the heart sounds were also heard distinctly; no other physical sign to be detected, except a stridulous breath sound, which had its locale evidently below the fourchette of the sternum, and which became more audible as the man became more hurried by the exertion of dressing and undressing. It now appeared to me sufficiently clear that there was an intra-thoracic tumour, but of what description-whether eneurismal or cancerous-it was as yet difficult to say; but, of course, the probabilities were in favour of the former. I stated my opinion to the patient's brother, and partly to himself, mentioning candidly my altered views of the case, and expressing my conviction that hopes of recovery could no longer be held out. I regret that I had no further opportunities of studying this interesting case during life, as the patient was again placed under his former attendant, at whose instance Dr. Noble was called in, and who, I understand, expressed his conviction that some aneurismal affection was present, and that the lungs were healthy; in this diagnosis differing from the ordinary attendant, who, up to the period of the patient's death, maintained the case to be phthisis. The man now grew rapidly worse, and symptoms of hydrops pericardii (as I am informed) set in, under which he sunk.

Having been made aware of his death, I succeeded, with much difficulty, in procuring a post mortem examinationa farour denied to his own attendant; and, assisted by my friend Mr. Murphy, demonstrator of anatomy in the Manchester School of Medicine-Dr. Noble and other friends being present-I made the inspection forty-eight hours after death. For sake of brevity, I will merely say that the lungs and pleura were perfectly healthy; that the pericardium contained fully six ounces of serum, but no lymph; and that the heart and its valves were quite free from disease. At the distance of an inch from the semilunar valves, and springing from the posterior wall of the aorta, there was a false aneurism of that vessel, as large as a small orange: the opening into it was very peculiar; it was lozenge-shaped, or, as a gentleman present observed, like the ace of diamonds; its longest diameter-the longitudinal -might be three-quarters of an inch; the edges were clean, $2 s$ if cut with an instrument; there was hardly any cavity, for the sac was filled with concentric lamellæ of firm fibrin; the posterior part of the tumour rested and pressed upon the front of the trachea. There was a smaller aneurism close to the arch, not larger than a nut, with a similar opening, but, of course, much smaller. Around the orifices the lining membrane of the ressel was thickened, but there was no atheromatous deposit; towards the valves it was quite healthy, and the mechanical action of these structures was perfect.

REMarks. The feature of special interest in the foregoing case-and for the value of which I am induced to publish it-is the light which the history appears to me to throw upon the origin of the disease. I suppose there can bardly be a doubt that the lesion of the aorta was the im- mediate effect of the violent occurrence which took place twelve months before the man's death. In the clinical history of thoracic aneurism, violent muscular effort has been always regarded as holding an important place in the causation of this malady; and hence the athletic exercises of the chase, boating, etc., are laid down as predisposing causes; and by way of illustration, such cases as that of the late Mr. Liston are in the habit of being quoted. But I am very doubtful whether muscular effort alone can suffice for the production of this terrible complaint. If such were the case, surely it would be much more common than it is. Where there is already a predisposition to the disease, extraordinary muscular effort may, unquestionably, prove an exciting cause; but to produce such a remarkable effect in a healthy subject, I consider the presence of another agent necessary ; and in those cases which are pointed out as confirmatory of the above opinion, such agency has always been present. I allude to the great mental excitement which invariably accompanies these unusual muscular actions. In the present instance, the man made the most strenuous exertion to free himself from his assailants; he was, at the same time, in a state of the highest degree of passion, so that the two conditions which I consider most favourable to the production of a physical injury to the main bloodvessel were present. And do not the excitement and exertion of the chase, cricket, and boating, supply precisely the same conditions?

That in this case, too, the disease was not the result of slow arteritis, the appearance of the opening I think sufficiently proves; for it presented none of the signs of ulceration or erosion, but was just what might be expected from a longitudinal crack taking place in the inner coat of the ressel, and expanding in a lateral direction.

Another interesting point in this case was the absence of what are considered by some the indispensable concomitants of aneurism, namely, impulse and bruit de souffet. The position of the tumour, and its solid contents, sufficiently explain the absence of impulse; might the shape of the opening, and its cleanly cut edges, account for the nonproduction of the abnormal sounds? The double sound, or pseudo heart sound, is considered by Dr. O'B. Bellingham the normal sound of aneurism; and such was heard over the spot where dulness was discovered; but I am not sure that what was heard did not proceed from the heart itself, as any solid body in the same locality would afford the same sounds, that is, convey them from the heart.

It is worthy of remark, also, the trifling amount of stridor observed, notwithstanding the evident pressure made by the tumour upon the front of the trachea; but, as Dr. Stokes observes, "A small aneurism, causing lateral compression of the trachea, will sooner produce stridor than a larger tumour, the pressure of which is more directly from before backwards." It should be borne in mind that stridulous breathing, in connexion with these aneurismal tumours, may proceed from two different sources: either from direct pressure upon the trachea, or one of its divisions, or from irritation of the nerves, which supply the muscles of the larynx. The last named distinguished physician has called the latter, "stridor from above", the former "stridor from below", and the distinction is very important. When the trachea is compressed within the thorax the stridor is generally persistent, though liable to considerable increase when the patient is excited; and the sound can be clearly perceived to proceed from below the fourchette of the sternum; and as a symptom, it does not indicate immediate danger. In the other case, the stridor is always paroxysmal, and its locality is easily referred to the upper part of the windpipe; it is a symptom of the most alarming import. I had an opportunity some time ago of witnessing a well marked case of this kind. The patient, a young man, suffered from attacks of dyspncea, with loud laryngeal stridor, at times so severe as to threaten immediate suffocation, and in a fit of which he eventually died. On dissection, there was found an aneurism, of the size of a filbert, springing from the under part of the arch of the aorta, just where the left recurrent nerve hooks round 
that reseel. The laryngeal affoction caused the true native of the disease to be suspected during life, although no corroborative evidence existed.

\section{ON THE CLOSURE OF ARTERIES AT THEIR ORIGIN ; AND ON SOME MORBID CHANGES OF THE HEART.}

By Thouas. Hodgrin, M.D.

[Real before the Hurreian Society, Narch $6 t h, 18 j 6$.]

Ar the kind invitation of my friend Dr. Ridge, I am again induced to occupy the attention of my fellow members of the Harveian Society; and, on the present occasion, I am indebted to him for the suggestion of the subject on which I am about to speak.

In the Hunterian Oration, which Dr. Ridge lately delivered, he has given a most valuable and elaborate analysis of the record of the symptoms, death, and post mortem exmination, of the great anatomist in whose honour the oration was pronounced. Angina pectoris and the pathological changes upon which it depends, form, of course, a prominent part of this interesting discourse. Nevertheless, Dr. Ridge felt himself so restricted by the time reasonably to be occupied in the delivery, that he was precluded from stating much which was present in his mind to offer. Amongst the points thus omitted, was the modus operandi of the closure of the coronary arteries. Knowing this was a subject to which I some years since paid distinct attention, he proposed it to me as one on which I might write a short article, to be laid before you. I do so with the more pleasure, as it recalls to my recollection two important communications made to this society at a time when I was 2 more frequent attendant at your meetings than I have lately had the adrantage of being. I allude to the cases of obliteration of the coronary arteries, presented by Dr. Ridge himself, and to the experiments of another distinguished member, John Erichsen, in which the coronary arteries were tied at the suggestion of Professor Sharpey, in both of which papers the importance of these arteries to the performance of the heart's functions was clearly shown.

In the cases related by Dr. Ridge in particular, I noticed at the time, as I believe he had also done himself, the operation of a principle which I had taught when I was in the practice of delivering lectures on morbid anatomy at Guy's Hospital. My attention to this point in the pathology of arteries was first excited on the occasion of the late Chas. A. Key operating on a patient affected with aneurism of the arteria innowinata.

He applied the ligature to the right carotid, and the patient rather suddenly expired a few hours after. On examination, the left carotid, though apparently healthy, and of normal size, was found to communicate with the arch of the aorta by an aperture scarcely large enough to admit the passage of a small ordinary probe. This con. formation of the artery, which very satisfaciorily accounted for the patient's death, by the deficient supply of blood which it caused the brain to receive, appeared at the time to be regarded merely as an untoward accidental coincidence.

In reflecting upon it, however, I quickly perceived that it was only the natural consequence of a pathological principle of very general application, and on which I had laid particular stress when treatiug of the serous membranes, which, on many accounts, afford the best field for the study of morbid processes in their simplest as well as their most evident forms. The principle to which I refer, is the contraction of the more or less plastic deposit resulting from inflammation. The deposit bencath the lining membrane of an artery forms no exception to this rule, but the contraction is probably the more marked in proportion 29 the deposit possesses the plastic character. It is extremely probable that the aneurismatic dilation takes place most readily at the very time at which the inflammatury process is producing the deposit, the artery then being in a more jielding state.

The branches given off from tho dilated ressel do not necessarily increase with the trunk from which they proceed, although this is sometimes the case, for which exemption an explanation may be given, which I shall presently offer. When the dilation of the trunk has acquired a considerable size, we might expect to find the branch baving a funnel-shaped mouth; which, however, I do not remember ever to have seen, unless the branch also has been diseased.

Let us now consider what must be the effect produced upon the mouth of the branch when the adrentitious deposit has arrived at that stage at which its contraction takes place.

We know that this contractile force is very great, insomuch, that muscular effort is incapable of resisting it ; as in the case of false membranes on the pleura costalis and diaphragm, and in the contraction of the cicatrices of burns. In the case of a dilated diseased artery the force of the heart in propelling the blood may for a time resist this contraction; but it must ultimately fail, and the contraction take place, more especially if the natural decline of the patient's strength or the course of treatment employed should have the effect of diminishing the force of the heart and the volume of the blood. Now, if by this contraction an area of an inch in diameter around the mouth of an arterial branch be reduced by only one-third of its diameter, it must have the effect of greatly diminishing or closing the mouth of the branch. When this idea had struck me, from seeing the specimen taken from $C$. Aston Key's patient, I sought its confirmation in other analogous specimens, and found that my riews were conspletely corroborated.

It must not be supposed that the process which I have described invariably takes place. As I have already observed, the branches may partake of the disease of the trunk, and, like it, become dilated and somewhat funnelshaped, in which case they may participate in the contraction also without being obliterated.

I have said that the integrity of the branches originating from a discased trunk has its analogy in the phenomena presented by the serous membranes. I allude to the abrupt and defined termination of inflammation in these membranes, which is sometimes seen where a sudden change of direction takes place: as, for example, in the pleura pulmonalis, in which it often ceases where the pleura passes from the external surface into the interlobular fissure, and also in many instances in which the peritoneum changes its direction.

Even where the branch may not participate in the disease of the trunk, its closure or contraction may be prevented by a further stage, into which the adventitious deposit may pass. Neither the atheromatous nor the bony. deposit, so commonly met with beneath the lining membrane in diseased arteries, is likely to undergo the process of contraction. It is, however, to this latter change to which I more particularly allude, as the comparatively salutary barrier by which nature obviates the inconreniences which must follow the obliteration of arterial branches at their mouths; and I have in my mind's cye preparations, in which this provision for the preservation of the circulation is strikingly exhibited in the human subject, and also in the horse, in which an aortic aneurism has become completely ossified, forming a permanent cavity of bone, with patent orifices for its branches.

Although by this provision the closure of the branches, given off from a discased trunk may be prevented from taking place through the contraction of the deposit situated beneath the lining membranc, it is quite possible for the obstruction of the blood from eutering the branch to be brought about by another morbid process taking its rise at this stage of the derangement. The lining membrane may give way and expose the osseous, or rather earthy, matter to immediate contact with the blood, which then begins to deposit phosphate of lime, or other earthy salts, upon the earthy surface so exposed. Such deposi- 